\title{
Optical code division multiple access codes comparison in free space optics and optical fiber transmission medium
}

\begin{abstract}
Performance of three different Optical Code Division Multiple Access codes namely Prime Code (PC), Quadratic Congruence (QC), and Khazani-Syed (KS) code are compared in Free Space Optic (FSO) and optical fiber transmission. The simulation results show that efficiency of a code family is medium-dependent. While one code family performs the best in fiber medium, it is not necessary that it acts the same in FSO. For instance, KS with code weight of 6 provides BER 10-12 at $600 \mathrm{~m}$ distance, while other codes cannot even reach to the threshold 10-9 at this point. However this code shows vulnerability against fiber dispersion. At $5 \mathrm{~km}$ fiber, it provides BER of 10-7, even less then PC with 10-8. In that point KS code with weight 6 attains the best performance with BER of 10-11.
\end{abstract}

Keyword: Coding; Dispersion; Free space optic (FSO); Optical code division multiple access (OCDMA); Optical fiber styling 